\title{
Development of human lung induction models for air pollutants' toxicity assessment
}

Shuyu Liu ${ }^{1,2}$, Renjun Yang ${ }^{1,2}$, Yongjiu Chen ${ }^{1,2}$, Xingchen Zhao ${ }^{1,2}$, Shaokun Chen ${ }^{1,2}$, Xuezhi Yang ${ }^{1,2}$, Zhanwen Cheng ${ }^{1,2}$, Bowen $\mathrm{Hu}^{1,2}$, Xiaoxing Liang ${ }^{1,2}$, Nuoya Yin ${ }^{1,2}$, Qian Liu ${ }^{1,2}$, Hailin Wang ${ }^{1,2}$, Sijin Liu $^{1,2}$, Francesco Faiola ${ }^{1,2, *}$

${ }^{1}$ State Key Laboratory of Environmental Chemistry and Ecotoxicology, Research Center for EcoEnvironmental Sciences, Chinese Academy of Sciences, Beijing, 100085, China

${ }^{2}$ College of Resources and Environment, University of Chinese Academy of Sciences, Beijing, 100049, China

*Corresponding Author: Francesco Faiola, E-mail: faiola@rcees.ac.cn, Tel/Fax: 86 10-62917609

Keywords: human pluripotent stem cells; alveolar type 2 epithelial cells; $\mathrm{BaP}$; nano carbon black; nano $\mathrm{SiO}_{2}$

\section{Supporting Information}

\section{Table of Content}

\begin{tabular}{|l|l|}
\hline Supporting Methods & S2 \\
\hline Table S1. RT-qPCR primer sequences & $\mathbf{S 4}$ \\
\hline Table S2. Antibodies for immunostaining and western blot & $\mathbf{S 4}$ \\
\hline Figure S1. Heatmap of the differentially expressed genes (DEGs) for H9 hESCs and & $\mathbf{S 6}$ \\
ATL cells & \\
\hline Figure S2. Derived ATLs exposed to $10 \mathrm{nM} \mathrm{BaP} \mathrm{for} \mathrm{7} \mathrm{days} \mathrm{did} \mathrm{not} \mathrm{show} \mathrm{significant}$ & $\mathbf{S 7}$ \\
difference in TGF $\beta / S M A D$ signaling activity & \\
\hline Figure S3. Characterization of nano carbon black and nano $\mathrm{SiO}_{2}$ & $\mathbf{S 7}$ \\
\hline Figure S4. Toxicity assays for nano carbon black on derived hLPs and ATLs & $\mathbf{S 8}$ \\
\hline Figure S5. Application of ATL organoids for nano $\mathrm{SiO}_{2}$ toxicity tests & $\mathbf{S 1 0}$ \\
\hline
\end{tabular}


AlamarBlue cell viability assay

Cells were incubated, after thorough washes with PBS, with $10 \mu \mathrm{M}$ AlamarBlue (Sigma-Aldrich, R7017) in DMEM/F12 at $37^{\circ} \mathrm{C}$ for $2 \mathrm{~h}$. Fluorescence values were measured with an excitation wavelength of $530 \mathrm{~nm}$ and an emission wavelength of $590 \mathrm{~nm}$ with a Varioskan LUX multimode microplate reader (ThermoFisher Scientific, VLBLATD2).

Intracellular reactive oxygen species (ROS) production measurement Intracellular ROS generation was detected with the 2,7-dichlorofluorescin diacetate (DCFH-DA) probe (Sigma-Aldrich, USA, D6883). Briefly, after 14 days of alveolar induction (without any treatment), ATLs were incubated with $10 \mu \mathrm{M}$ DCFH-DA at $37^{\circ} \mathrm{C}$ for $30 \mathrm{~min}$. Then, cells were treated with indicated doses of $\mathrm{BaP}$ and nanoparticles (or DMSO vehicle control), for $6 \mathrm{~h}$. Fluorescence values were measured with an excitation wavelength of $488 \mathrm{~nm}$ and an emission wavelength of $525 \mathrm{~nm}$, in a Varioskan LUX multimode microplate reader.

Quantitative real-time reverse transcription polymerase chain reaction (RT-qPCR) analysis Total RNA was extracted with TRIzol reagent (Life Technologies, 15596018). Reverse transcription was performed using TAKARA RT MasterMix (RR036A), and qPCR was executed with TAKARA SYBR Green PreMix (RR042A), on a QuantStudio 6 Flex System (Life Technologies). Each qPCR assay was performed in triplicate. GAPDH and $\beta$-ACTIN were used as reference genes. A list of the primer sets used is provided in Table S1.

RNA-seq analysis

RNA samples were extracted with a Qiagen RNeasy Mini kit (Qiagen, 74104). RNA-seq analyses were completed at Beijing Genomics Institute (BGI). Raw data accession number: GSE152854 and GSM4473070-1 (for H9 hESC control). Qualified RNA samples (assessed with an Agilent 2100 instrument and Agilent RNA 6000 nano Reagents Part 1) were sequenced with a BGISEQ-500 platform. Kyoto Encyclopedia of Genes and Genomes (KEGG) annotations were conducted at BGI. Briefly, gene 
numbers were computed for every term and the DEGs were subjected to a hypergeometric test. Significantly enriched KEGG pathways were determined based on a q-value $\leq 0.05$ after false discovery rate correction.

Western blot

Protein samples were collected with RIPA buffer (Solarbio, r0020) supplemented with a proteinase and phosphatase inhibitor cocktail. After protein quantification with a BCA (bicinchoninic acid) kit (Beyotime, p0009), equal amounts of proteins were subjected to SDS-PAGE. Then, proteins were electro-transferred from the acrylamide gels onto nitrocellulose membranes (BIORAD, 1620112). Membranes were blocked with 5\% BSA (Beyotime, st023). Primary antibody incubation was performed overnight at $4^{\circ} \mathrm{C}$. An HRP-linked goat anti-rabbit antibody (Cell Signaling Technology, 7074) was used to recognize primary antibodies for $2 \mathrm{~h}$ at room temperature. Membranes were imaged with a ChemiDoc Touch imaging system (BIO-RAD, USA) after treatment with a Super ECL Detection Reagent (YEASEN, 36208ES60). Information on the primary antibodies used is provided in Table S2.

Immunofluorescence staining

Monolayer cultured cells were fixed with 4\% paraformaldehyde (PFA)/PBS for $10 \mathrm{~min}$ at room temperature. After thorough wash in PBS, cells were blocked with 5\% goat serum (Solarbio, SL038)/0.1\% Triton X-100 (Amresco, 0694-1L)/PBS for $1 \mathrm{~h}$ at $4^{\circ} \mathrm{C}$. Primary antibody incubation was performed overnight at $4{ }^{\circ} \mathrm{C}$ followed by secondary antibody incubation for $1 \mathrm{~h}$ at room temperature. 3D cultured cells were fixed within Matrigel with $4 \%$ PFA/PBS for 20 min at $4{ }^{\circ} \mathrm{C}$, and blocked as in monolayer conditions. Then, 3D cultured cells were incubated with primary antibodies for $48 \mathrm{~h}$ at $4{ }^{\circ} \mathrm{C}$ and followed by secondary antibody incubation overnight. In both conditions, primary antibodies were diluted in $1 \%$ bovine serum albumin (BSA)/0.1\% Triton X-100/PBS. Secondary antibodies were diluted in 5\% goat serum/1\% BSA/0.1\% Triton X-100/PBS. Cells were stained with $10 \mu \mathrm{g} / \mathrm{mL}$ DAPI (Solarbio, C0065) at room temperature for $15 \mathrm{~min}$. The antibodies used are listed in Table S2.

Table S1. RT-qPCR primer sequences 


\begin{tabular}{|c|c|c|}
\hline Target & Forward primer & Reverse primer \\
\hline GAPDH & GGTCACCAGGGCTGCTTTTA & GGATCTCGCTCCTGGAAGATG \\
\hline$\beta-A C T I N$ & ACTCTTCCAGCCTTCCTTCC & TCTCCTTCTGCATCCTGTCG \\
\hline NKX2.1 & CTCATGTTCATGCCGCTC & GACACCATGAGGAACAGCG \\
\hline ID2 & GACAGCAAAGCACTGTGTGG & TCAGCACTTAAAAGATTCCGTG \\
\hline SOX9 & ATGAAGATGACCGACGAGCA & AACTTGTCCTCCTCGCTCTC \\
\hline SOX2 & GCTTAGCCTCGTCGATGAAC & AACCCCAAGATGCACAACTC \\
\hline AXIN2 & CAGAGAGAAATGCATCGCAGTGT & TCTCCAACTCCAGCTTCAGCTTT \\
\hline SFTPC & AGCAAAGAGGTCCTGATGGA & CGATAAGAAGGCGTTTCAGG \\
\hline$L A M P 3$ & TATTCACTGCCACCCACCAT & TCTGACCGGTTGTGCTTTTG \\
\hline$A B C A 3$ & AAGATGTAGCGGACGAGAGG & CCCCGGTCAGCATTTTGAAA \\
\hline LPCAT1 & TGAAGTGTGTTGCAGAACCG & ACAGAGACTCGAAACCAGGG \\
\hline NAPSA & ACGCCTCCACAAAACTTCAC & TGGCAAACTTGGTCCCATTG \\
\hline KI67 & TCTTCAGAATGGAAGGAAGTCAACTG & GGCCTTGGAATCTTGAGCTTTCT \\
\hline
\end{tabular}

Table S2. Antibodies for immunostaining and western blot

\begin{tabular}{lllll}
\hline Antibody & Company & Cat no. & Species & Dilution \\
NKX2.1 & Cell Signaling Technology & 12373 & rabbit & $1: 200$ \\
SOX2 & Cell Signaling Technology & $3597 \mathrm{~S}$ & rabbit & $1: 200$ \\
E-CADHERIN & Invitrogen & $13-1900$ & rat & $1: 1000$ \\
B-CATENIN & Cell Signaling Technology & 8480 & rabbit & $1: 2000$ \\
pro-SPC & Abcam & & & \\
GAPDH & Abcam & 90716 & rabbit & $1: 100$ \\
& & 128915 & rabbit & $1: 5000$ \\
\hline
\end{tabular}




\begin{tabular}{|c|c|c|c|c|c|}
\hline pSMAD2 & & Cell Signaling Technology & 3108 & rabbit & $1: 1000$ \\
\hline SMAD2 & & Cell Signaling Technology & 5339 & rabbit & $1: 1000$ \\
\hline $\begin{array}{l}\text { Alex Fluor488 } \\
\text { anti-rabbit }\end{array}$ & Goat & Cell Signaling Technology & $4340 \mathrm{~S}$ & Goat & $1: 1000$ \\
\hline $\begin{array}{l}\text { HRP-linked } \\
\text { anti-rabbit }\end{array}$ & goat & Cell Signaling Technology & 7074 & Goat & $1: 2000$ \\
\hline $\begin{array}{l}\text { Alex Fluor647 } \\
\text { anti-rat }\end{array}$ & Goat & Invitrogen & A-21248 & Goat & $1: 2000$ \\
\hline
\end{tabular}




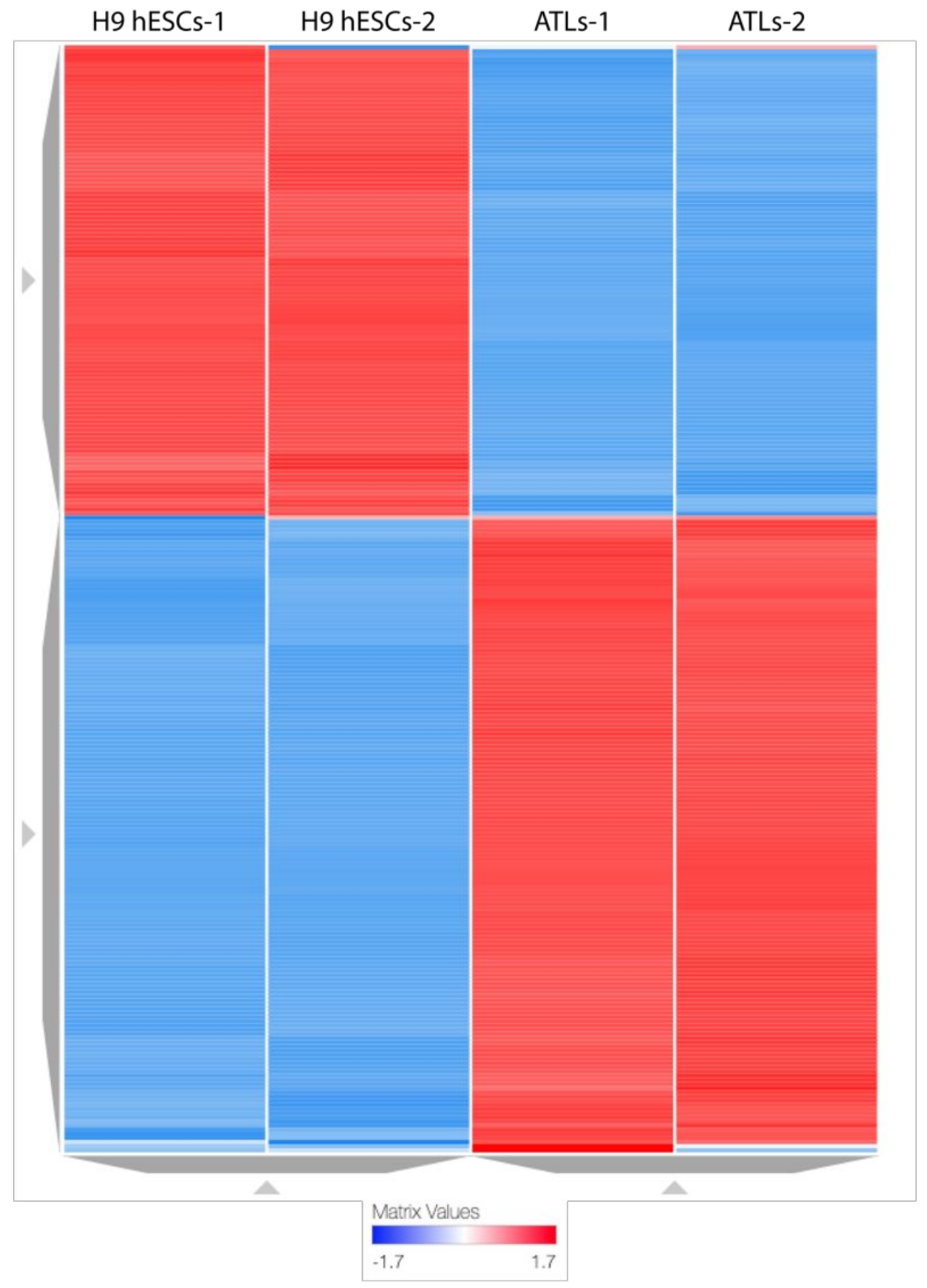

Figure S1. Heatmap of the differentially expressed genes (DEGs) for H9 hESCs and ATL cells. 


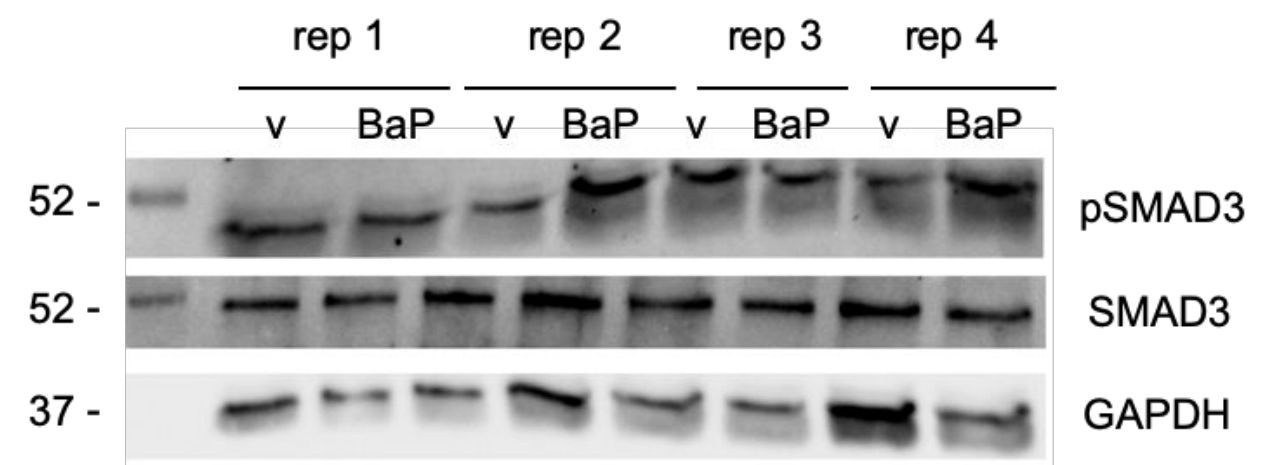

Figure S2. Derived ATLs exposed to $10 \mathrm{nM}$ BaP for 7 days did not show significant difference in TGF $\beta /$ SMAD signaling activity. Shown are western blot assays for phosphorylated (p) SMAD3, and total SMAD3 protein levels. Four different experiments (reps 1-4) were performed. GAPDH was used as a loading control.

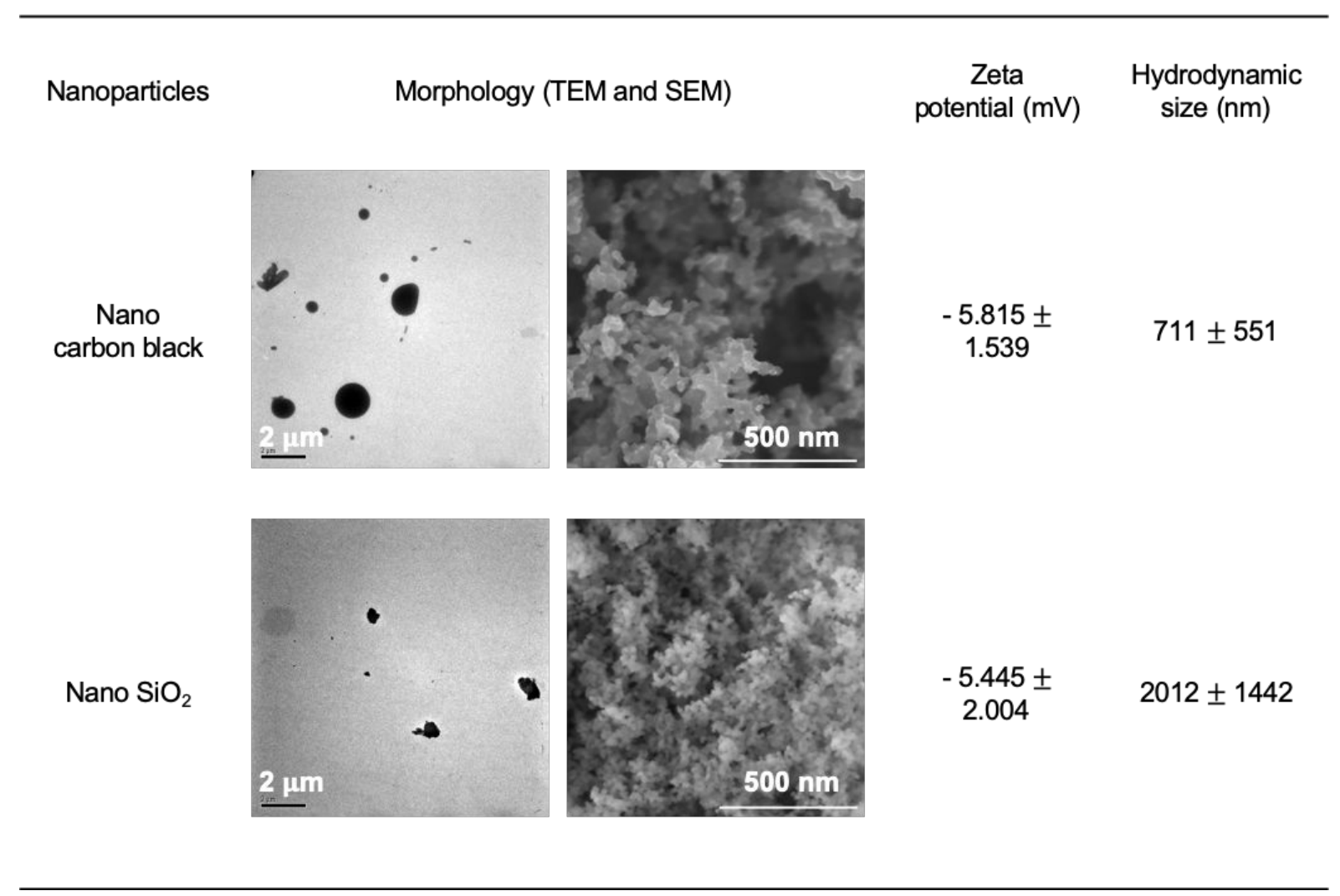

Figure S3. Characterization of nano carbon black and nano $\mathrm{SiO}_{2}$. Shown are representative TEM and SEM images, with indicated scale bars, and values for zeta potential and hydrodynamic size. 
A

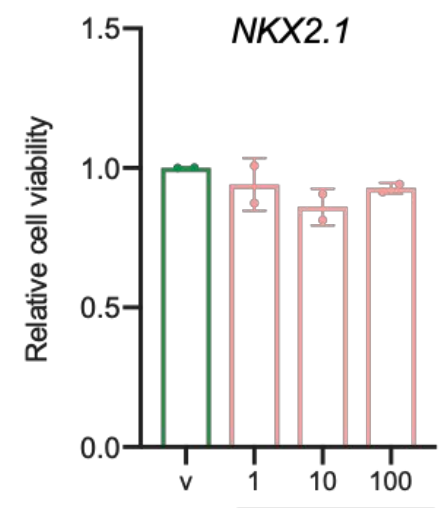

ID2

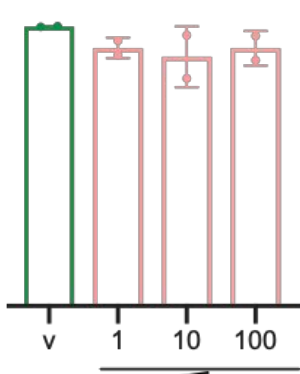

B

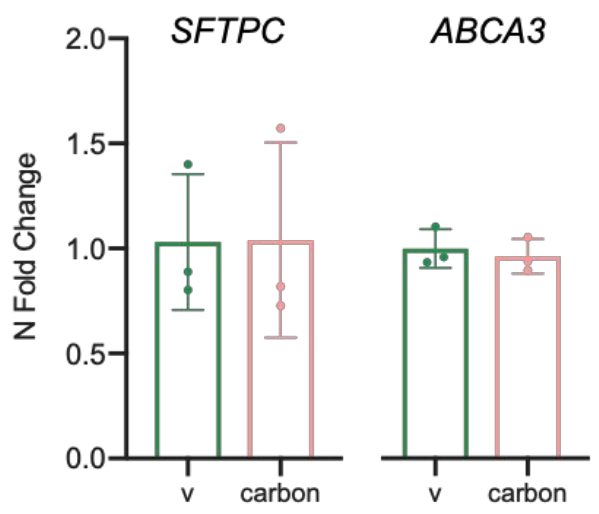

carbon black $\mathrm{ng} / \mathrm{mL}$

C

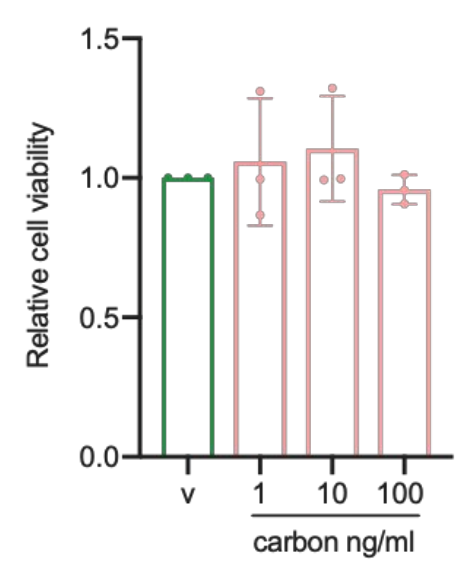

$\mathrm{D}$

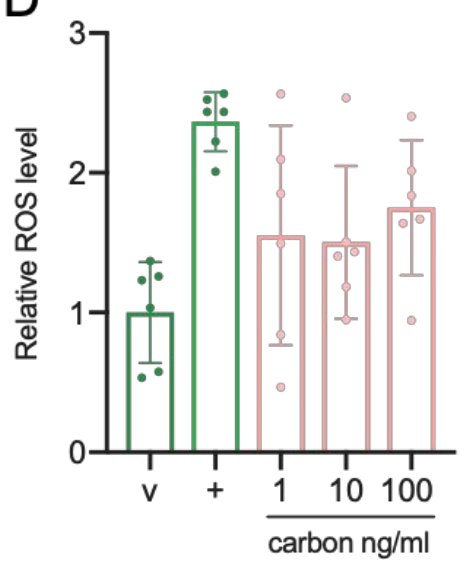

Figure S4. Toxicity assays for nano carbon black on derived hLPs and ATLs.

A. Nano carbon black exposure during hLP induction from hPSCs did not affect NKX2.1 and ID2 expression in hLPs. Shown are RT-qPCR results of three independent biological experiments. Genes were normalized to GAPDH and comparisons were made with the vehicle control (v) set as 1 .

B. $100 \mathrm{ng} / \mathrm{mL}$ nano carbon black exposure during ATL induction from hLPs did not affect SFTPC and $A B C A 3$ expression in ATLs. Shown are RT-qPCR results of three independent biological experiments. Genes were normalized to GAPDH and comparisons were made with the vehicle control.

C. Nano carbon black exposure to ATLs for 7 days did not cause significant cytotoxicity. Results of three independent biological experiments are shown. Normalization was made to the vehicle control (set as 1) in each biological experiment. Comparisons were made between vehicle and treated groups. 
D. Nano carbon black exposure to ATLs for $6 \mathrm{~h}$ did not consistently induce significant increase in ROS generation. Results of three independent biological experiments are shown. Normalization was made to the vehicle control. "+" stands for the positive control provided in the kit. 
A
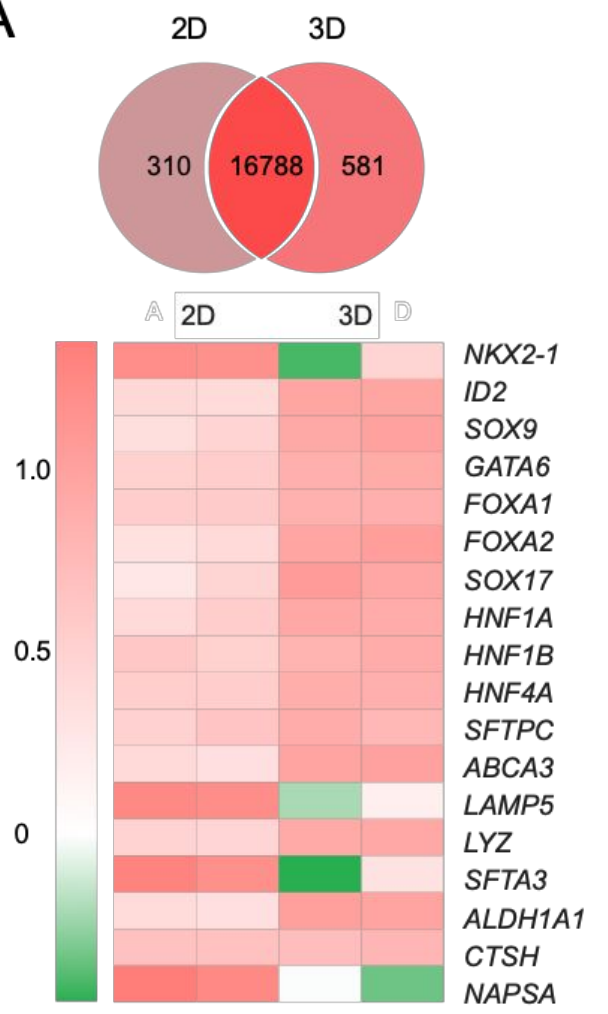

C

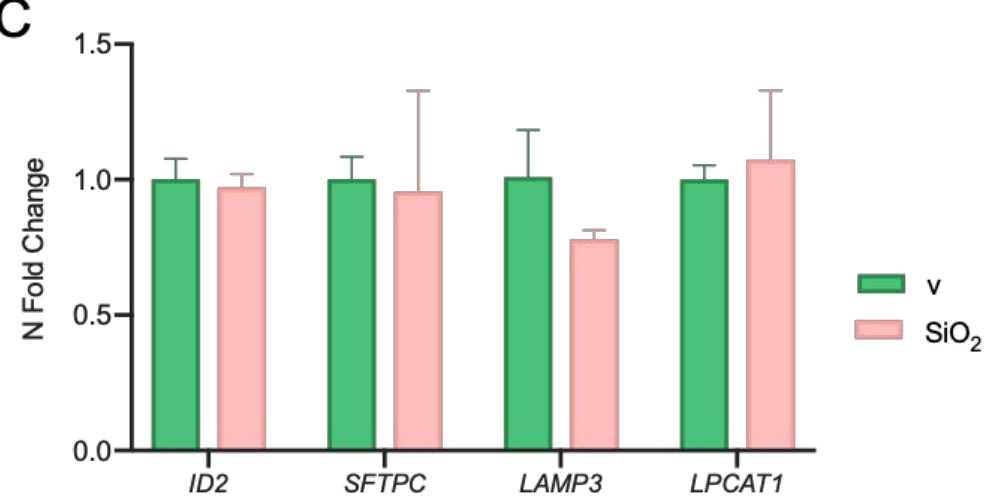

E

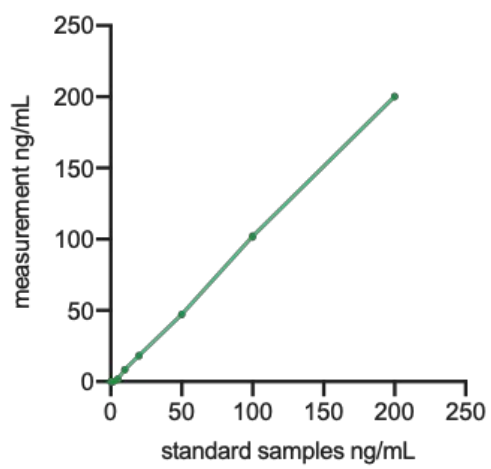

B
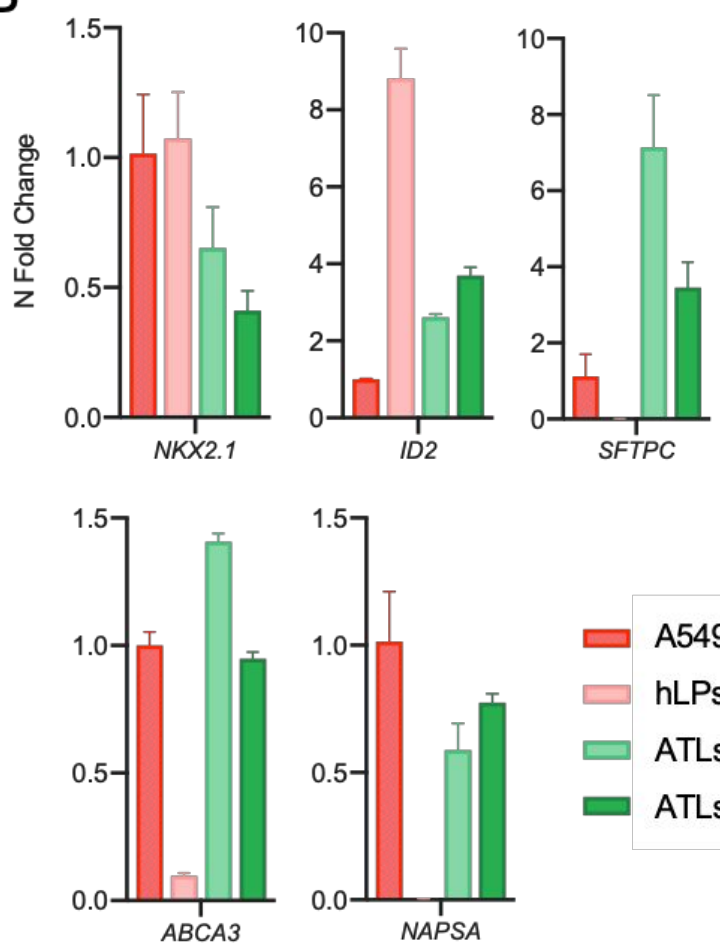

A549

hLPs

ATLs d7

ATLsd14

D

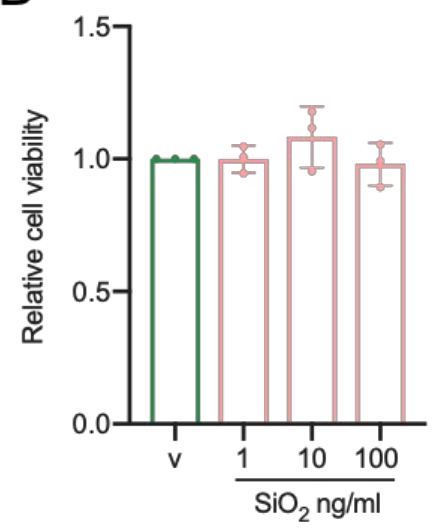

Figure S5. Application of ATL organoids for nano $\mathrm{SiO}_{2}$ toxicity tests. 
A. ATLs derived from monolayer (2D) induction and in organoid culture conditions (3D) did not differ significantly in their global gene expression. Shown are the Venn diagram for DEGs between the 2 groups (top) and gene expression comparison, in duplicate, for a set of lung marker genes between 2D and 3D ATLs (bottom).

B. RT-qPCR gene expression comparisons among A549, hLPs, d7 ATLs, and d14 ATLs. Shown are the averages from three independent biological experiments. Genes were normalized to GAPDH and the values in $\mathrm{A} 549$ set as $1 ; * P<0.05$.

C. Nano $\mathrm{SiO}_{2}$ treatment for 14 days did not affect Matrigel-based ATL organoid viability. Results of three independent biological experiments are shown. Normalization was made to the vehicle control in each biological experiment. Comparisons were made between vehicle and treated groups, ${ }^{*} P<0.05$. D. Nano $\mathrm{SiO}_{2}$ treatment for 14 days did not affect AT2 marker gene expression in Matrigel-based ATL organoids. RT-qPCR results for three independent biological experiments are shown. Genes were normalized to GAPDH and comparisons were made with the vehicle control (v), set as 1 .

E. ICP-MS calibration curve of silicon element. Defined concentrations of silicon standard solution were measured before sample analyses. The measurement for each concentration was done once. 